\section{The risk factors and prognostic implication of acute pulmonary edema in resuscitated cardiac arrest patients}

\author{
Dae-hyun Kang ${ }^{1 *}$, Joonghee Kim ${ }^{1 *}$, Joong Eui Rhee ${ }^{2}$, Taeyun Kim', \\ Kyuseok Kim ${ }^{1}$, You Hwan Jo ${ }^{1}$, Jin Hee Lee ${ }^{1}$, Jae Hyuk Lee ${ }^{1}$, Yu Jin Kim', \\ Seung Sik Hwang ${ }^{3}$ \\ 'Department of Emergency Medicine, Seoul National University Bundang Hospital, Seongnam, Korea \\ ${ }^{2}$ Department of Emergency Medicine, Samsung Medical Center, Sungkyunkwan University School of \\ Medicine, Seoul, Korea \\ ${ }^{3}$ Department of Social and Preventive Medicine, Inha University School of Medicine, Incheon, Korea
}

Objective Pulmonary edema is frequently observed after a successful resuscitation in out-ofhospital cardiac arrest (OHCA) patients. Currently, its risk factors and prognostic implications are mostly unknown.

Methods Adult OHCA patients with a presumed cardiac etiology who achieved sustained return of spontaneous circulation (ROSC) in emergency department were retrospectively analyzed. The patients were grouped according to the severity of consolidation on their initial chest X-ray (group I, no consolidation; group II, patchy consolidations; group III, consolidation involving an entire lobe; group IV, total white-out of any lung). The primary objective was to identify the risk factors of developing severe pulmonary edema (group III or IV). The secondary objective was to evaluate the association between long-term prognosis and the severity of pulmonary edema.

Results One hundred and seven patients were included. Total duration of cardiopulmonary resuscitation $(\mathrm{CPR})$ and initial $\mathrm{pCO}_{2}$ level were both independent predictors of developing severe pulmonary edema with their odds ratio $(\mathrm{OR})$ being 1.02 (95\% confidence interval $[\mathrm{Cl}], 1.00$ to 1.04; per 1 minute) and $1.04(95 \% \mathrm{Cl}, 1.01$ to 1.07 ; per $1 \mathrm{mmHg})$, respectively. The long term prognosis was significantly poor in patients with severe pulmonary edema with a OR for good outcome (6-month cerebral performance category 1 or 2) being $0.22(95 \% \mathrm{Cl}, 0.06$ to 0.79$)$ in group III and $0.16\left(95 \% \mathrm{Cl}_{1} 0.04\right.$ to 0.63$)$ in group IV compared to group I.

Conclusion The duration of $\mathrm{CPR}$ and initial $\mathrm{pCO}_{2}$ level were both independent predictors for the development of severe pulmonary edema after resuscitation in emergency department. The severity of the pulmonary edema was significantly associated with long-term outcome.

Keywords Pulmonary edema; Cardiopulmonary resuscitation; Ventilation; Prognosis

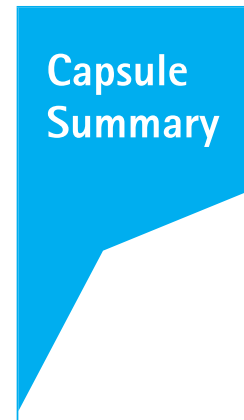

What is already known

Acute pulmonary edema is commonly observed after prolonged chest compressions. Previous studies have suggested that pulmonary edema results largely from the acutely increased pulmonary intravascular pressure generated by closed chest compressions.

What is new in the current study

The severity of pulmonary edema seen after return of spontaneous circulation is significantly associated with long-term survival and neurologic outcomes. Prolonged chest compressions and increased initial $\mathrm{pCO}_{2}$ level are both independent risk factors for the development of acute pulmonary edema.
elSSN: 2383-4625

Received: 2 July 2014

Revised: 29 July 2014

Accepted: 7 August 2014

Correspondence to: Joong Eui Rhee Department of Emergency Medicine, Samsung Medical Center,

Sungkyunkwan University School of Medicine, 81 Irwon-ro, Gangnam-gu, Seoul 135-710, Korea

E-mail: rheeje@gmail.com

*The first two authors contributed equally to this study.

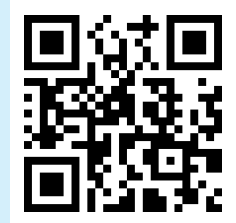

How to cite this article:

Kang Dh, Kim J, Rhee JE, Kim T, Kim K, Jo YH, Lee JH, Lee JH, Kim YJ, Hwang SS. The risk factors and prognostic implication of acute pulmonary edema in resuscitated cardiac arrest patients. Clin Exp Emer Med 2015;2(1):110-116.

This is an Open Access article distributed under the terms of the Creative Commons Attribution Non-Commercial License (http:// creativecommons.org/licenses/by-nc/3.0/). 


\section{INTRODUCTION}

Pulmonary edema is a complication that develops during or shortly after cardiopulmonary resuscitation (CPR).$^{1-8}$ Its vivid manifestations, including both pink frothy secretion during chest compressions and multiple consolidation shadows on postresuscitation chest X-rays, are common in cardiac arrest patients. Studies have suggested that pulmonary edema results largely from the acutely increased pulmonary intravascular pressure generated by closed chest compressions, ${ }^{7}$ and therefore rapidly resolves after successful resuscitation. ${ }^{6}$ However, there has been relatively little investigation to further clarify the causes and consequences of pulmonary edema. Currently, it is unknown whether any non-hemodynamic factors, such as systemic inflammation or coagulopathy, influence development of pulmonary edema. Another important information gap is whether pulmonary edema affects prognosis. Therefore, the primary objective of this study was to identify factors associated with the severity of pulmonary edema, other than hemodynamic variables, in resuscitated out-of-hospital cardiac arrest (OHCA) patients. The secondary objective was to assess its prognostic significance.

\section{METHODS}

\section{Study design}

This is a single-center retrospective study of consecutive patients suspected to have had OHCA due to cardiac etiology from 2008 to 2012. The primary objective was to identify factors associated with the severity of pulmonary edema seen after return of spontaneous circulation (ROSC). The secondary objective was to assess the association between severity of the pulmonary edema and long-term prognosis. The institutional review board of the study facility approved the study and provided a waiver of consent.

\section{Study setting and population}

The study was conducted in a 950-bed teaching hospital located in a city with an estimated population of 1,000,000. Emergency medical services were provided by the governmental fire services, and the level of prehospital CPR was restricted to basic life support. ${ }^{9}$ Advanced cardiovascular life support (based on American Heart Association CPR guidelines) was provided in the emergency department (ED) after arrival. Blood sampling was routinely performed on arrival via peripheral vein or femoral puncture. Chest radiographs were routinely obtained after ROSC.

Adult (aged 18 or older) OHCA patients from 2008 to 2012 with a sustained ROSC after an unexpected sudden cardiac arrest were included (Table 1). We excluded patients with prehospital ROSC, and those who did not have a chest radiographs taken within 3 hours of ED arrival, because of the tendency for pulmonary edema to rapidly disappear after ROSC. ${ }^{6}$

\section{ED OHCA registry}

The researchers have maintained a registry of all consecutive OHCA patients transferred to the study facility since $2008 .^{10,11} \mathrm{It}$ includes prehospital Utstein-style elements, ED CPR events, and patient outcomes. The Utstein-style elements and CPR events are collected by emergency medical technicians (EMTs) working in the ED, and then reviewed and refined by the ED CPR researchers. Patient outcomes, including survival and 6-month cerebral performance category (CPC) scales, ${ }^{12}$ are assessed by review of electronic medical records and by monthly telephone interviews performed by fourth-year emergency medicine (EM) residents.

Table 1. Definition of terms and detailed criteria used in this study

\begin{tabular}{|c|c|}
\hline Term & Definition \\
\hline Public location & $\begin{array}{l}\text { Street, park, beach or public buildings such as shopping center, sports facility, entertainment center, airport, railway station, church or } \\
\text { office building. Cardiac arrest occurred in nursing home facility or ambulance was categorized as non-public location in this study. }\end{array}$ \\
\hline Witnessed cardiac arrest & Cardiac arrest witnessed by another person or monitored by EMTs. \\
\hline Bystander CPR & Any resuscitative effort accompanied by chest compression. Rescue breathing-only effort was excluded. \\
\hline Time to CPR & Time interval between first recognition of cardiac arrest, witnessed or not, and first attempt of chest compression. \\
\hline Total duration of CPR & Time interval between first attempt of chest compression and first documented ROSC regardless of its duration. \\
\hline Initial rhythm & The first rhythm documented by EMTs or ED physician. \\
\hline Presumed cardiac aetiology & $\begin{array}{l}\text { Witnessed sudden collapse suggestive of sudden cardiac death or unwitnessed cardiac arrest without any documented evidence of } \\
\text { conditions that might be significantly related with the cardiac arrest such as underlying acute infection, trauma, pulmonary embolism, } \\
\text { advanced malignancy, exacerbation of underlying disease, major stroke or bed-bound status. }\end{array}$ \\
\hline Sustained ROSC & Documented evidence of return of spontaneous circulation sustained longer than 20 minutes. \\
\hline Survival discharge & $\begin{array}{l}\text { The term "survival discharge" indicates that the patient was discharged to home or long-term care facility, regardless of his or her } \\
\text { functional status. }\end{array}$ \\
\hline Good neurologic outcome & 6-month cerebral performance category score 1-2. \\
\hline
\end{tabular}

EMT, emergency medical technician; CPR, cardiopulmonary resuscitation; ROSC, return of spontaneous circulation; ED, emergency department. 

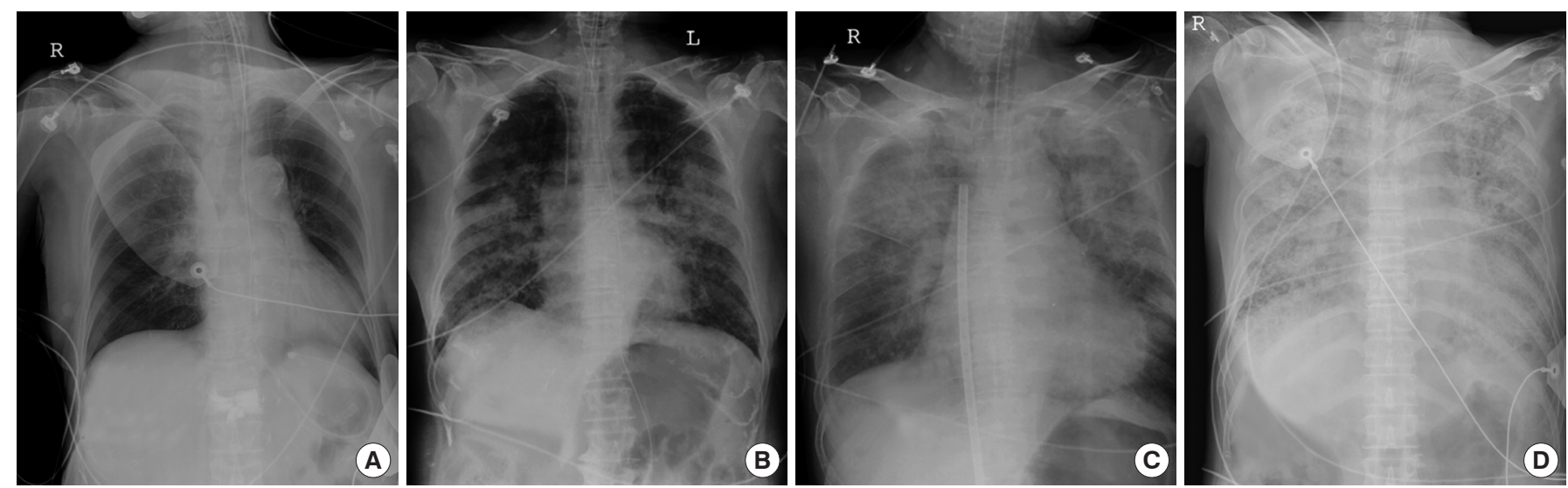

Fig. 1. A plain chest X-ray (anteroposterior view) showing the distribution and extent of consolidation in each of the four groups of acute pulmonary edema by severity: (A) group I, no consolidation; (B) group II, alveolar pattern; (C) group III, lobar pattern; (D) group IV, entire lung.

\section{Study protocol and measurements}

Utstein-style elements and demographic variables for the included patients were obtained from the registry. Laboratory values were obtained from their medical records. Severity of pulmonary edema and the degree of underlying cardiomegaly were assessed from the initial post-ROSC chest radiograph (anteroposterior [AP] view). The severity of pulmonary edema was graded using the following procedure: each patient's initial chest radiograph (AP view) was downloaded from the picture archiving and communication system (PACS) server and merged into a single Microsoft PowerPoint file. After de-identification and random shuffling, the severity of acute pulmonary edema was graded by an EM physician blinded to patient outcomes using criteria developed by the authors. Those criteria are as follows: group I (no consolidation), normal image or redistribution or interstitial pattern change; group II (alveolar pattern), patchy consolidations without involvement of an entire lobe; group III (lobar pattern), any consolidation involving more than one entire lobe; group IV (entire lung), total whiteout of more than one entire lung (Fig. 1, Table 2). The degree of cardiomegaly present (cardiothoracic ratio) was measured by another EM physician in a separate session after re-shuffling of the images.

\section{Data analysis}

Spearman's rho was used to assess the strength of correlation and its level of significance. Multivariate logistic regression using backward elimination was used (likelihood ratio, elimination threshold $P>0.1$ ) to identify independent risk factors for development of severe pulmonary edema (group III and IV). Both univariate and multivariate logistic regression analyses were performed to assess the association between severity of pulmonary edema and long-term patient outcomes. The goodness of fit and the area
Table 2. Description of the system for grading the severity of pulmonary edema

\begin{tabular}{lc}
\hline Pulmonary edema severity grading & Description of the consolidation \\
\hline I (no consolidation) & $\begin{array}{c}\text { Normal image or redistribution or } \\
\text { interstitial pattern change }\end{array}$ \\
II (alveolar pattern) & $\begin{array}{c}\text { Patchy consolidations without } \\
\text { involvement of an entire lobe }\end{array}$ \\
III (lobar pattern) & $\begin{array}{c}\text { Any consolidation involving more } \\
\text { than one entire lobe }\end{array}$ \\
IV (entire lung) & Total white-out of more than one \\
& entire lung \\
\hline
\end{tabular}

under the receiver operator characteristic curve were calculated to determine the model fit and discrimination of the final model. The results of the logistic regression analysis are presented as odds ratios (ORs) and their 95\% confidence intervals (Cls). P-values $<0.05$ were considered significant. All analyses were performed using STATA ver. 12 (StataCorp LP, College Station, TX, USA).

\section{RESULTS}

From 2008 to 2012, 608 adult OHCA patients received advanced cardiovascular life support in the ED, with 278 of them achieving sustained ROSC. After exclusion of patients with presumed noncardiac etiology $(n=158)$ and those with no chest radiographs taken within 3 hours of ED arrival $(n=13), 107$ patients were included in the study population (Fig. 2). Baseline characteristics of the study population are summarized in Table 3. Among the study population, 78 patients (72.9\%) were male, and the median age was 69 (interquartile range, 55 to 75). A total of 43 patients (40.2\%) survived, and were discharged to go home or to a nursing care facility. Overall, 31 patients (29\%) had a favorable long-term outcome (6-month $\mathrm{CPC}, 1$ or 2$)$. 


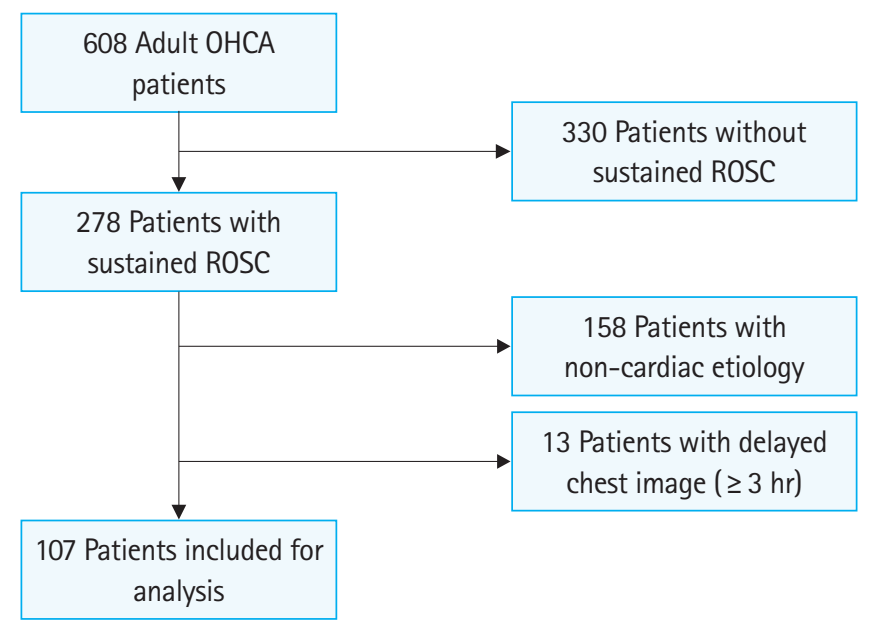

Fig. 2. Flowchart for patient selection. OHCA, out-of-hospital cardiac arrest; ROSC, return of spontaneous circulation.
Table 3. Characteristics of the study population $(n=106)$

\begin{tabular}{ll}
\hline Characteristic & No. (\%) \\
\hline Sex (male) & $78(72.9)$ \\
Age (yr), median (IQR) & $69(55-75)$ \\
Public place & $35(32.7)$ \\
Shockable rhythm & $48(44.9)$ \\
Witnessed cardiac arrest & $86(80.4)$ \\
Bystander CPR & $47(43.9)$ \\
Time to CPR (min), median (IQR) & $4(0-10)$ \\
Total duration of CPR (min), median (IOR) & $23(15-34)$ \\
Therapeutic hypothermia & $60(56.1)$ \\
Survival discharge & $43(40.2)$ \\
Six-month CPC 1 or 2 & $31(29.0)$
\end{tabular}

IQR, interquartile range; $C P R$, cardiopulmonary resuscitation; $C P C$, cerebral performance category.

Table 4. Patient characteristics stratified according to the severity of pulmonary edema

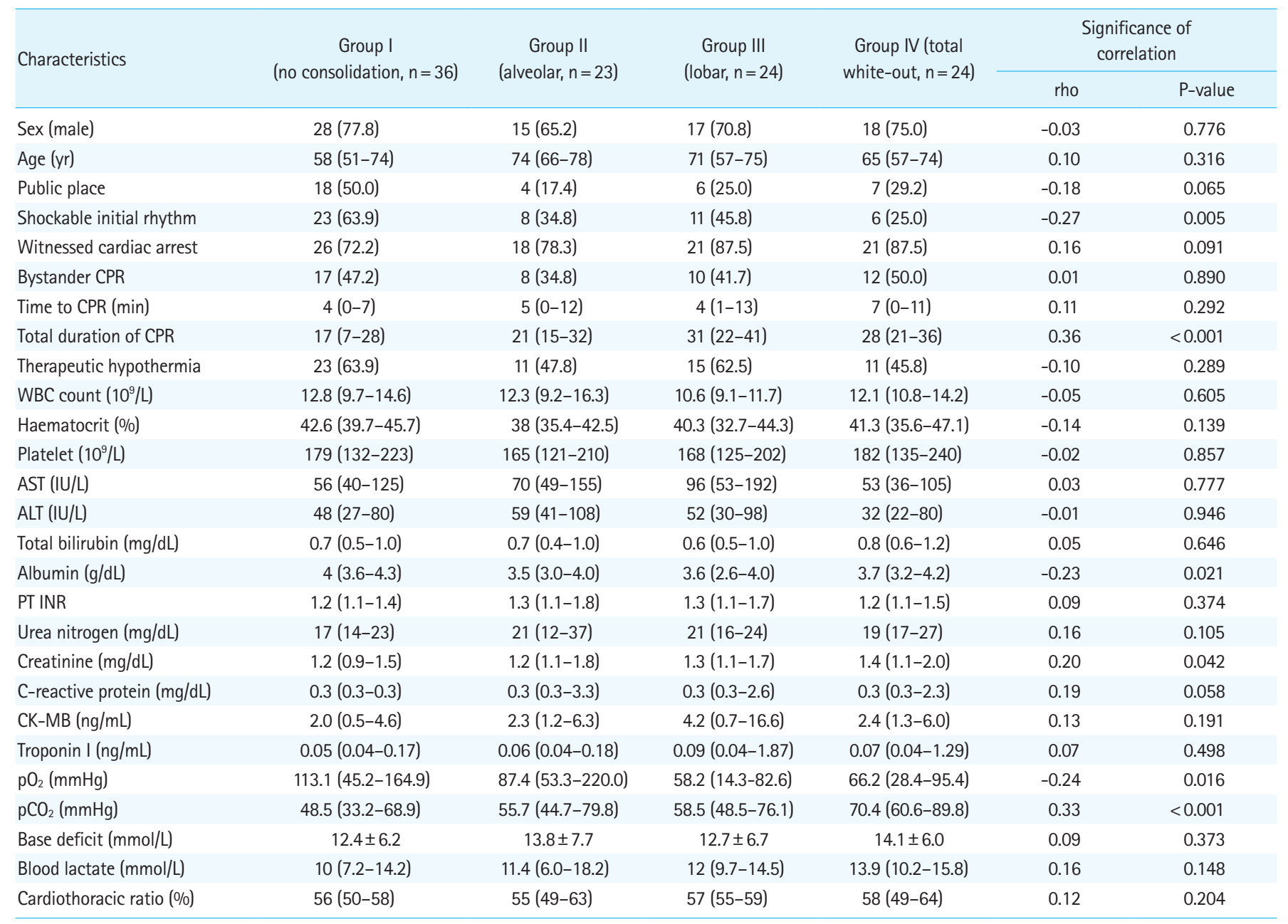

Values are presented as number (\%), median (IQR) or mean \pm SD.

CPR, cardiopulmonary resuscitation; WBC, white blood cell; AST, aspartate aminotransferase; ALT, alanine aminotransferase; PT, prothrombin time; INR, international normalisation ratio; $\mathrm{CK}$, creatinine kinase. 
Patients' baseline characteristics and initial laboratory variables were stratified according to the severity of pulmonary edema (Table 4). A shockable initial rhythm, total duration of CPR (sum of pre-hospital and ER CPR duration), serum albumin and creatinine levels, and initial $\mathrm{pO}_{2}$ and $\mathrm{pCO}_{2}$ levels were all significantly correlated with severity of pulmonary edema (Spearman's rho: $-0.27,0.36,-0.23,0.20,-0.24$, and 0.33 , respectively; P-values: 0.005 , <0.001, 0.021, 0.042, 0.016, and <0.001, respectively). These variables were used to construct a multivariate logistic regression model using backward elimination (likelihood ratio, elimination threshold $P>0.1$ ) to identify the independent risk factors for developing severe pulmonary edema (group III and IV) (Table 5). The model showed that total duration of $\mathrm{CPR}$ and initial $\mathrm{pCO}_{2}$ level were both independent risk factors for the development of severe pulmonary edema (total duration of CPR: OR, 1.02 per one minute; $95 \% \mathrm{Cl}, 1.00$ to $1.04 ; \mathrm{pCO}_{2}$ : OR, 1.04 per $1 \mathrm{mmHg} ; 95 \%$ $\mathrm{Cl}, 1.01$ to 1.07$)$.

Table 6 displays the association between severity of pulmonary edema and long-term patient outcomes. In univariate logistic regression analysis, increased severity of pulmonary edema was sig-

Table 5. Univariate logistic regression analysis and backward stepwise logistic regression model for prediction of the severity of pulmonary edema

\begin{tabular}{lcc}
\hline Variable & Odds ratio (95\% Cl) & P-value \\
\hline Univariate logistic regression ${ }^{\mathrm{a}}$ & & \\
Shockable initial rhythm & $0.50(0.23-1.08)$ & 0.078 \\
Total duration of CPR $(/ \mathrm{min})$ & $1.05(1.02-1.08)$ & 0.002 \\
Albumin $(/ \mathrm{g} / \mathrm{dL})$ & $0.51(0.28-0.93)$ & 0.027 \\
$\mathrm{Creatinine} / \mathrm{mg} / \mathrm{dL})$ & $1.52(0.99-2.32)$ & 0.054 \\
$\mathrm{pO}_{2}(/ \mathrm{mmHg})$ & $1.00(0.99-1.00)$ & 0.028 \\
$\mathrm{pCO}_{2}(/ \mathrm{mmHg})$ & $1.03(1.01-1.04)$ & 0.006 \\
$\mathrm{Backward} \mathrm{stepwise} \mathrm{logistic} \mathrm{regression} \mathrm{model}^{\mathrm{b})}$ & & \\
$\mathrm{pCO}_{2}(/ \mathrm{mmHg})$ & $1.02(1.00-1.04)$ & 0.038 \\
Total duration of CPR $(/ \mathrm{min})$ & $1.04(1.01-1.07)$ & 0.016 \\
\hline
\end{tabular}

$\mathrm{Cl}$, confidence interval; $\mathrm{CPR}$, cardiopulmonary resuscitation.

a) Variables significantly correlated with pulmonary edema severity (Table 4). ${ }^{\text {b) }} 101$ patients (94.4\%) were included in the model. P-value for goodness of fit (chi-squared) and the area under the receiver operator characteristic curve were 0.430 and 0.72 , respectively. nificantly associated with poor long-term outcomes. The odds ratios of patients surviving to discharge in groups III and IV relative to group I were $0.29(95 \% \mathrm{Cl}, 0.10$ to 0.89$)$ and $0.24(95 \% \mathrm{Cl}, 0.08$ to 0.74$)$, respectively. The odds ratios of achieving favorable neurologic outcomes in groups III and IV relative to group I were 0.22 ( $95 \% \mathrm{Cl}, 0.06$ to 0.79 ) and 0.16 ( $95 \% \mathrm{Cl}, 0.04$ to 0.63$)$. Similar associations were also observed after adjusting for application of therapeutic hypothermia.

\section{DISCUSSION}

In this study, we found that prolonged chest compressions and a high initial $\mathrm{pCO}_{2}$ level are both independent risk factors for development of pulmonary edema. We also found that severity of pulmonary edema significantly correlates with both patient survival and long-term neurologic outcomes.

The association of prolonged chest compressions with severity of pulmonary edema, which was not evident in the previous study by Dohi, ${ }^{3}$ is hardly surprising. If high pulmonary intravascular pressure due to closed chest compressions is the main mechanism for development of pulmonary edema as previously suggested, ${ }^{7}$ then prolongation of chest compressions should lead to increased net extravasation of intravascular contents. The relative homogeneity of the etiology of cardiac arrest, and the larger sample size compared to the study by Dohi, ${ }^{3}$ could have helped us identify this association. However, it is prudent to consider that a reverse causal relationship might exist; that is, patients who develop acute pulmonary edema are more resistant to $C P R$, which might lead to delayed ROSC in these patients. On a similar note, the higher $\mathrm{pCO}_{2}$ level in patients with severe pulmonary edema could just be an early sign of developing pulmonary edema that becomes more evident in postresuscitation chest images. Another less plausible explanation is that effective ventilation during CPR exerts a protective effect by counteracting fluid leakage from pulmonary capillaries.

Most of the variables that we originally assumed would have a

Table 6. Association between severity of pulmonary edema and long-term patient outcomes

\begin{tabular}{|c|c|c|c|c|c|c|c|c|c|c|}
\hline \multirow{3}{*}{ Group } & \multicolumn{5}{|c|}{ Survival discharge } & \multicolumn{5}{|c|}{ Good neurologic outcome } \\
\hline & \multirow{2}{*}{ No. $(\%)$} & \multicolumn{2}{|c|}{ Unadjusted } & \multicolumn{2}{|c|}{ Adjusted for TH } & \multirow{2}{*}{ No. (\%) } & \multicolumn{2}{|c|}{ Unadjusted } & \multicolumn{2}{|c|}{ Adjusted for TH } \\
\hline & & OR $(95 \% \mathrm{Cl})$ & P-value & OR $(95 \% \mathrm{Cl})$ & P-value & & OR $(95 \% \mathrm{Cl})$ & P-value & OR $(95 \% \mathrm{Cl})$ & P-value \\
\hline I & $21(58.3)$ & 1.00 (reference) & & 1.00 (reference) & & $17(47.2)$ & 1.00 (reference) & & 1.00 (reference) & \\
\hline ॥ & $9(39.1)$ & $0.46(0.16-1.34)$ & 0.153 & $0.47(0.16-1.39)$ & 0.173 & $7(30.4)$ & $0.49(0.16-1.47)$ & 0.204 & $0.47(0.15-1.44)$ & 0.185 \\
\hline III & $7(29.2)$ & $0.29(0.10-0.89)$ & 0.029 & $0.29(0.10-0.89)$ & 0.030 & 4 (16.7) & $0.22(0.06-0.79)$ & 0.020 & $0.22(0.06-0.78)$ & 0.019 \\
\hline IV & $6(25.0)$ & $0.24(0.08-0.74)$ & 0.013 & $0.25(0.08-0.77)$ & 0.016 & $3(12.5)$ & $0.16(0.04-0.63)$ & 0.009 & $0.15(0.04-0.61)$ & 0.008 \\
\hline P-trend & & & 0.006 & & 0.007 & & & 0.002 & & 0.002 \\
\hline
\end{tabular}

$\mathrm{TH}$, therapeutic hypothermia; $\mathrm{OR}$, odds ratio; $\mathrm{Cl}$, confidence interval. 
significant association with the severity of pulmonary edema showed no association. Because of the possibility of increased vascular permeability from global ischemic injuries, ${ }^{13-15}$ we expected that radiologic changes indicative of pulmonary edema would be more severe if surrogate markers of ischemic burden, such as initial lactate level or base deficit, were increased. We also expected the presence of initial coagulopathy would be associated with radiologic changes because we thought some portion of such changes would be due to pulmonary hemorrhage caused by vigorous chest compressions. ${ }^{16-19}$ However, none of these variables showed an association with the severity of pulmonary edema. Only serum albumin level, which is the major contributor to serum oncotic pressure, was significantly associated with development of pulmonary edema. Although serum albumin level was not included in the final stepwise logistic regression, we presume that albumin might have a protective effect against development of pulmonary edema by decreasing the net osmotic pressure gradient across the pulmonary vascular wall. A future study analyzing the composition of tracheal secretions collected during CPR may provide a more definitive answer to this question. The absence of an association between the cardiothoracic ratio and severity of pulmonary edema may indicate that pulmonary edema in CPR is mostly determined by the chest compressions rather than the size or function of the heart.

In this study, the severity of acute pulmonary edema was significantly correlated with both survival to discharge and the 6-month neurologic outcome. These correlations would be due to differences in baseline characteristics, such as the initial electrocardiography rhythm. However, the development of pulmonary edema could have a detrimental effect of its own. In addition to causing pulmonary gas exchange abnormalities, thereby decreasing the effectiveness of $\mathrm{CPR}_{1}^{2,3,20-22}$ pulmonary edema can increase pulmonary vascular resistance, ${ }_{1}^{23}$ and thus further impair systemic circulation during CPR, which is an extreme low flow state. ${ }^{24}$ Coupled with poor gas exchange, the low cardiac output during CPR would be a "worst case" scenario for both the heart and brain.

This study has several limitations. First, the results of this study are based on a retrospective analysis of an ED OHCA registry and medical records. Thus, biases intrinsic to such a study design are unavoidable. Second, we used multivariate logistic regression with backward elimination to identify independent risk factors for development of acute pulmonary edema. This can underestimate the importance of certain combinations of variables, and has the potential to identify spurious associations. ${ }^{25}$ Although the two variables left in the final model also showed a strong correlation with the severity of acute pulmonary edema, as assessed by Spearman's rho and therefore the final model was also predictable, we could not exclude such possibilities. Third, the author-developed criteria used for grading severity of acute pulmonary edema have not previously been tested. However, we presume that the simplicity of the criteria and their close correlation with long-term prognoses can support their validity. Fourth, it is possible that a significant portion of haziness that was interpreted as "pulmonary edema" could have been "pulmonary contusion" from chest compression. Future research on how fast these conditions resolve after successful resuscitation might shed light on this question.

In conclusion, prolonged chest compressions and increased initial $\mathrm{pCO}_{2}$ level are both independent risk factors for the development of acute pulmonary edema in patients with sudden cardiac arrest. As the severity of pulmonary edema is significantly associated with long-term survival and neurologic outcomes, future research on how to prevent or reverse its development, especially in prolonged CPR, may provide a means to improve resuscitation outcomes.

\section{CONFLICT OF INTEREST}

No potential conflict of interest relevant to this article was reported.

\section{ACKNOWLEDGMENTS}

This work was supported by the SNUBH research fund (grant no. 02-2013-100).

\section{REFERENCES}

1. Neumar RW, Nolan JP, Adrie C, et al. Post-cardiac arrest syndrome: epidemiology, pathophysiology, treatment, and prognostication. A consensus statement from the International Liaison Committee on Resuscitation (American Heart Association, Australian and New Zealand Council on Resuscitation, European Resuscitation Council, Heart and Stroke Foundation of Canada, InterAmerican Heart Foundation, Resuscitation Council of Asia, and the Resuscitation Council of Southern Africa); the American Heart Association Emergency Cardiovascular Care Committee; the Council on Cardiovascular Surgery and Anesthesia; the Council on Cardiopulmonary, Perioperative, and Critical Care; the Council on Clinical Cardiology; and the Stroke Council. Circulation 2008;118:2452-83.

2. Fillmore SJ, Shapiro M, Killip T. Serial blood gas studies during cardiopulmonary resuscitation. Ann Intern Med 1970;72:465-9.

3. Dohi S. Postcardiopulmonary resuscitation pulmonary edema. Crit Care Med 1983;11:434-7. 
4. Nagel EL, Fine EG, Krischer JP, Davis JH. Complications of CPR. Crit Care Med 1981;9:424.

5. Ornato JP, Bryson BL, Donovan PJ, Farquharson RR, Jaeger C. Measurement of ventilation during cardiopulmonary resuscitation. Crit Care Med 1983;11:79-82.

6. Ornato JP, Ryschon TW, Gonzalez ER, Bredthauer JL. Rapid change in pulmonary vascular hemodynamics with pulmonary edema during cardiopulmonary resuscitation. Am J Emerg Med 1985;3:137-42.

7. Ornato JP. Hemodynamic monitoring during CPR. Ann Emerg Med 1993;22:289-95.

8. Davis K Jr, Johannigman JA, Johnson RC Jr, Branson RD. Lung compliance following cardiac arrest. Acad Emerg Med 1995; 2:874-8.

9. Ro YS, Shin SD, Song KJ, et al. A comparison of outcomes of out-of-hospital cardiac arrest with non-cardiac etiology between emergency departments with low- and high-resuscitation case volume. Resuscitation 2012;83:855-61.

10. Kim J, Choi BS, Kim K, et al. Prognostic performance of diffusion-weighted MRI combined with NSE in comatose cardiac arrest survivors treated with mild hypothermia. Neurocrit Care 2012;17:412-20.

11. Kim J, Kim K, Lee JH, et al. Prognostic implication of initial coagulopathy in out-of-hospital cardiac arrest. Resuscitation 2013;84:48-53.

12. Booth $C M$, Boone RH, Tomlinson G, Detsky AS. Is this patient dead, vegetative, or severely neurologically impaired? Assessing outcome for comatose survivors of cardiac arrest. JAMA 2004;291:870-9.

13. Asimakopoulos G, Smith PL, Ratnatunga CP, Taylor KM. Lung injury and acute respiratory distress syndrome after cardiopulmonary bypass. Ann Thorac Surg 1999;68:1107-15.

14. Clark SC. Lung injury after cardiopulmonary bypass. Perfusion 2006;21:225-8.
15. Apostolakis E, Filos KS, Koletsis E, Dougenis D. Lung dysfunction following cardiopulmonary bypass. J Card Surg 2010;25: 47-55.

16. Cho SH, Kim EY, Choi SJ, et al. Multidetector CT and radiographic findings of lung injuries secondary to cardiopulmonary resuscitation. Injury 2013;44:1204-7.

17. Tyburski JG, Collinge JD, Wilson RF, Eachempati SR. Pulmonary contusions: quantifying the lesions on chest $\mathrm{X}$-ray films and the factors affecting prognosis. J Trauma 1999;46:833-8.

18. Mateos Rodriguez AA, Pascual JM, Vallejo FP, Garcia AP, Belmonte AA. Lung injuries secondary to mechanical chest compressions. Resuscitation 2012;83:e203.

19. Perkins GD, Brace $S$, Gates S. Mechanical chest-compression devices: current and future roles. Curr Opin Crit Care 2010;16: 203-10.

20. Said SI, Longacher JW Jr, Davis RK, Banerjee CM, Davis WM, Wooddell WJ. Pulmonary gas exchange during induction of pulmonary edema in anesthetized dogs. J Appl Physiol 1964; 19:403-7.

21. Idris AH, Wenzel V, Becker LB, Banner MJ, Orban DJ. Does hypoxia or hypercarbia independently affect resuscitation from cardiac arrest? Chest 1995;108:522-8.

22. von Planta I, Weil MH, von Planta M, Gazmuri RJ, Duggal C. Hypercarbic acidosis reduces cardiac resuscitability. Crit Care Med 1991;19:1177-82.

23. West JB, Dollery CT, Heard BE. Increased pulmonary vascular resistance in the dependent zone of the isolated dog lung caused by perivascular edema. Circ Res 1965;17:191-206.

24. Hoeper MM, Galie N, Murali S, et al. Outcome after cardiopulmonary resuscitation in patients with pulmonary arterial hypertension. Am J Respir Crit Care Med 2002;165:341-4.

25. Livingston $E_{1}$ Cao J, Dimick JB. Tread carefully with stepwise regression. Arch Surg 2010;145:1039-40. 